\title{
Differential evolution based method for total transfer capability evaluation
}

\author{
R. Rajathy ${ }^{1 *}$, R. Gnanadass ${ }^{1}$, K. Manivannan ${ }^{1}$ and Harish Kumar ${ }^{2}$ \\ ${ }^{1}$ Department of Electrical and Electronics Engineering, Pondicherry Engineering College, Puducherry, INDIA, \\ ${ }^{2}$ Department of Physics, Pondicherry Engineering College, Puducherry, INDIA, \\ E-mail: (rajathy_harishkumar@rediffmail.com (R. Rajathy) *Corresponding Author)
}

\begin{abstract}
The application of Differential Evolution (DE) to compute the Total Transfer Capability (TTC) in deregulated market is proposed in this paper. The objective is to maximize a specific point-to-point power transaction without violating system constraints using DE. This algorithm is based on full ac optimal power flow solution to account for the effects of active and reactive power flow, voltage limits, and line flow limits. The real power output of generators in source area and the real power of the loads in the sink area were adjusted to obtain the maximum transfer capability. In order to calculate TTC more accurately, Transmission Reliability Margin is incorporated in the calculation of TTC by considering single and multiline outages and also a three phase to ground fault near a bus and clearing it by isolating the faulty line after certain time interval. The performance of the proposed method is tested on the modified IEEE-30 bus system and results are compared with that of Particle Swarm Optimization method (PSO). Further, the results are compared with other published results using CPF and EP. It is found that DE provides more reliable results than other methods.
\end{abstract}

Keywords: Total transfer capability, available transfer capability, transmission reliability margin, differential evolution, particle swarm optimization.

\section{Introduction}

Recently, there have been huge economic benefits to the society due to the deregulation of industries such as telecommunications and airlines. This stimulates the restructuring of electric power sector also. The present electrical utilities around the world are undergoing a radical transformation from an essentially regulated and monopolistic industry to a deregulated one. The deregulated electric industry is characterized by the competition in generation with guaranteed access to open transmission (NERC, 1995; NERC, 1999). Deregulation of power system is restructuring the power market so as to involve the private power producers in the system to supply powers. With the endeavor of achieving diversified customer choice of power suppliers and competitive electricity rates, vertically integrated utilities are broken down into generation, transmission and distribution entities. Transmission system, which acts as a common corridor between suppliers and customers, plays a significant role in the deregulated market structure.

In this scenario, there exists a technical challenge of finding the optimal balance between maximum power transfer capability and system stability margins. This is important as transfer capability values dominate critical decision - making of many power system operations and planning. All parties in this open access environment will try to procure the energy from the cheaper source for greater profit margins. This may lead to overloading of certain corridors of the transmission network. Consequently, the line flows, voltage and generation limits, will be violated affecting system security. Hence, utilities need to evaluate adequately the 'Total Transfer Capability (TTC)' to ensure that system reliability is maintained.

A wide variety of mathematical methods and algorithms have been developed for calculating TTC. These methods can be divided into four types as follows: Continuation Power Flow (CPF) method, Repetitive Power Flow (RPF) method, Linear Available Transfer Capability (LATC) method and Optimal Power Flow (OPF) based method.

The CPF method traces the power flow solution curve, starting at a base load, leading to the steady state voltage stability limit or the critical maximum loading point of power systems (Ejebe et al, 1998). This method can overcome the singularity of the Jacobian matrix near the saddle- node bifurcation point, or the critical point. However, the computational effort involved is large. Also, the timing requirement for determining TTC is severe. 
The RPF method, based on a generalized search method, repeatedly solves conventional power flow equations, where the successive power flow solutions are obtained to establish the maximum transfer capability (Gravener et al, 2000). In order to increase certain power, RPF uses a common loading factor for a cluster of generators and loads. This leads to conservative TTC, since the optimal distribution of generation and load are ignored.

The LATC method is based on linear incremental power flow approximation, which calculates Power Transfer Distribution Factors (PTDF) to determine the transfer capabilities of power system (Ejebe et al, 2000). This method is attractive because the factors are easy to calculate and can give rough figures of TTC quickly. However, the method is based on dc load flow, ignoring voltage and reactive power effects. This results in a stressed system with insufficient reactive power support and voltage control.

OPF method has been a powerful tool under very active development for over three decades. The objective functions of the OPF problem are generally non-linear and non-convex in nature. The traditional optimization approaches such as non-linear programming, quadratic programming, linear programming, mixed integer programming and interior point method (Dai et al, 2000) are used to solve the OPF problem. The literature on these approaches was reviewed by (Mamoh et al, 2000). The convergence of these methods depends upon the nature of the objective function. To overcome this difficulty, many heuristic search algorithms such as Genetic Algorithms (GA) (Bakritzis, 2002), Evolutionary Programming (EP) (Yuryevich et al, 1999), Simulated Annealing (SA) (Rao-sepulveda et al, 2003), Tabu search (Abido et al, 2002, Kulworawanichpong et al, 2002) have been proposed by many researchers to solve the OPF problem.

Recently, particle swarm optimization (PSO) (Kennedy et al, 1995; Park, 2005) and differential evolution (DE) (Price et al, 1996; 1999, Rajathy et al, 2009) have been introduced for solving real valued problems. Both techniques have shown great promise in several real world applications. In the present study, we used DE as an optimization tool to solve maximum power transfer optimal power flow problem in deregulated market. The applicability of the method is investigated for modified IEEE 30 bus system.

\section{Mathematical Modeling}

In this section, Total transfer capability problem is formulated as an optimal power flow problem. The objective function is to maximize the power that can be transferred from a specific set of generators in a source area to loads in a sink area, subject to the constraints of load flow equations and system operation limits. The sum of real power loads in the sink area at the maximum power transaction is defined as the TTC value.

The objective function for the OPF-based TTC calculation is expressed mathematically as

$$
\begin{gathered}
\text { MaxF }=\sum_{i=1}^{N D_{-} S N K} P_{D i} \\
P_{G i}-P_{D i}-\sum_{j=1}^{N} V_{i} V_{j} Y_{i j} \cos \left(\theta_{i j}-\delta_{i}+\delta_{j}\right)=0 \\
Q_{G i}-Q_{D i}+\sum_{j=1}^{N} V_{i} V_{j} Y_{i j} \operatorname{Sin}\left(\theta_{i j}-\delta_{i}+\delta_{j}\right)=0 \\
P_{G i}^{\min } \leq P_{G i} \leq P_{G i}^{\max } \quad i=1,2, \ldots \ldots, N G \\
Q_{G i}^{\min } \leq Q_{G i} \leq Q_{G i}^{\max } \quad i=1,2, \ldots \ldots ., N G \\
V_{i}^{\min } \leq V_{i} \leq V_{i}^{\max } \quad i=1,2, \ldots \ldots, N \\
\left|S_{L i}\right| \leq S_{L i}^{\max } \quad i=1,2, \ldots \ldots, N L
\end{gathered}
$$

where $F$ is the total load of load buses in a sink area. Further, $P_{G i}$ and $Q_{G i}$ are the real and reactive power generation at bus $i$. $P_{D i}$ and $Q_{D i}$ are the real and reactive load at bus $i, V_{i}$ and $V_{j}$ are the voltage magnitudes at bus $i$ and $j$ respectively. $Y_{i j}$ and $q_{\mathrm{ij}}$ are the magnitude and angle of element $i, j$ of bus admittance matrix. $\delta_{i}$ and $\delta_{j}$ are the voltage angles of bus $i$ and $j$. $P_{G i}^{\min }$ and $P_{G i}^{\max }$ are the lower and upper limits of real power generation at bus $i . Q_{G i}^{\min }$ and $Q_{G i}^{\max }$ are the lower and upper limits of reactive power generation at limits of voltage magnitude at bus $i .\left|S_{L i}\right|$ is the $i^{t h} \quad$ line or transformer loading and $S_{L i}^{\text {max }}$ is the $i^{\text {th }}$ line or transformer-loading limit. Here $N$ is the total number of buses, $N G$ is the number of generators, $N L$ is the number of branches and $N D_{-} S N K$ is the number of load buses in sink area. 


\section{Overview of Differential Evolution}

Differential Evolution is an optimization algorithm developed by Storn and Price, which solves real-valued problems based on the principles of natural evolution (Price, 1996; Price et al, 1999). DE uses a population $P$ of size $N_{p}$, composed of floating point encoded individuals that evolve over $\mathrm{G}$ generations to reach an optimal solution. Each individual $X_{i}$ is a vector that contains as many parameters as the problem decision variables $D$.

The population size $N_{p}$ is an algorithm control parameter selected by the user which remains constant throughout the optimization process.

$$
\begin{gathered}
P^{(G)}=\left[X_{i}^{(G)}, \ldots . ., X_{N_{P}}^{(G)}\right] \\
X_{i}^{(G)}=\left[X_{1, i}^{(G)}, \ldots, X_{D, i}^{(G)}\right]^{T}, \quad i=1, \ldots, N_{p}
\end{gathered}
$$

The optimization process in differential evolution is carried out with three basic operations viz, mutation, crossover and selection. This algorithm starts by creating an initial population of $N_{p}$ vectors. Random values are assigned to each decision parameter in every vector according to

$$
X_{j, i}^{(0)}=X_{j}^{\min }+\eta_{j}\left(X_{j}^{\max }-X_{j}^{\max }\right)
$$

where $i=1, \ldots, N_{p}$ and $j=1, \ldots . D ; X_{j}^{\min }$ and $X_{j}^{\max }$ are the lower and upper bounds of the $j^{\text {th }}$ decision parameter; and $\eta_{j}$ is an uniformly distributed random number within $[0,1]$ generated a new for each value of $j \cdot X_{j, i}^{(0)}$ is the $j^{\text {th }}$ parameter of the $i^{\text {th }}$ individual of the initial population.

The mutation operator creates mutant vectors $\left(X_{i}^{\prime}\right)$ by perturbing a randomly selected vector $\left(X_{a}\right)$ with the difference of two other randomly selected vectors $\left(X_{b}\right.$ and $\left.X_{c}\right)$,

$$
X_{i}^{\prime(G)}=X_{a}^{(G)}+F\left(X_{b}^{(G)}-X_{c}^{(G)}\right), \quad i=1 \ldots N_{P}
$$

where $X_{a}, X_{b}$ and $X_{c}$, are randomly chosen vectors $\in\left\{1, \ldots . . N_{p}\right\}$ and $a \neq b \neq c \neq i . X_{a}, X_{b}$ and $X_{c}$ are selected a new for each parent vector. The scaling constant $(F)$ is an algorithm control parameter used to control the perturbation size in the mutation operator and improve algorithm convergence.

The crossover operation generates trial vectors $\left(X_{i}^{\prime \prime}\right)$ by mixing the parameters of the mutant vectors with the target vectors $\left(X_{i}\right)$, according to a selected probability distribution,

$$
\begin{aligned}
& X_{j, i}^{\mathrm{\prime}(G)}=X_{j, i}^{\prime(G)} \text {, if } \eta_{j}^{\prime} \leq C_{R} \text { or } j=q \\
& \quad=X_{j, i}^{(G)} \text {, otherwise }
\end{aligned}
$$

where $i=1, \ldots, N_{p}$ and $j=1, \ldots . D ; q$ is a randomly chosen index $\in\left\{1, \ldots . . N_{p}\right\}$ that guarantees that the trial vector gets at least one parameter from the mutant vector; $\eta_{j}^{\prime}$ is a uniformly distributed random number within $[0,1]$ generated a new for each value of $j$. Crossover constant $C_{R}$ is an algorithm parameter that controls the diversity of the population and aids the algorithm to escape from local optima. $X_{j, i}^{(G)}, X_{j, i}^{\prime(G)}$ and $X_{j, i}^{\prime \prime(G)}$ are the $j^{\text {th }}$ parameter of the $i^{\text {th }}$ target vector, mutant vector, and trial vector at generation G, respectively. Finally, the selection operator determines the population by choosing between the trial vectors and their predecessors (target vectors) those individuals that present a better fitness or are more optimal.

$$
\begin{aligned}
X_{i}^{(G+1)} & =X_{i}^{\prime \prime(G)} \text { if } f\left(X_{i}^{\prime \prime(G)}\right) \leq f\left(X_{i}^{(G)}\right), \quad i=1, \ldots \ldots, N_{p} \\
& =X_{i}^{(G)}, \text { otherwise }
\end{aligned}
$$

The optimization process is repeated for several generations, allowing individuals to improve their fitness as they explore the solution space in the search for optimal values. 
DE offers several variants or strategies for optimization. These can be denoted by $\mathrm{DE} / \mathrm{x} / \mathrm{y} / \mathrm{z}$, where $\mathrm{x}$ refers to the vector used to generate mutant vectors, $\mathrm{y}$ the number of difference vectors used in the mutation process and $\mathrm{z}$ the crossover scheme used in the crossover operation. There are the ten different working strategies proposed by (Price et al, 1999). The working algorithm used in this paper is the seventh strategy of DE (i.e.) DE/rand/1/bin in which DE represents differential evolution, rand is any randomly chosen vector for perturbations, 1 represents the number of difference vectors to be perturbed and bin is the binomial type of crossover used.

\section{Solution Methodology}

The TTC from the source area to the sink area is calculated using Differential Evolution. While calculating TTC, we have incorporated contingencies such as single line and multiline outages and a three phase to ground fault. A base case ac power flow is established initially, in which the system load is supplied without violating the system constraints. Figure 1 shows the base case power flows in the transmission lines of the test system. The base case is modified by varying the generation in the source area and the load in sink area simultaneously. This process continues until the contingency causes the violation of any one of the system limits such as thermal limit, voltage limit and stability limit. The minimal amount of power transfer among all the contingencies under any one of this violation, is observed as the TTC for the corresponding power transaction which is given as

$$
T T C=\operatorname{Min}\left(T T C_{0}, T T C_{K}\right)
$$

where $T T C_{0}$ is the maximum amount of power transfer without contingency constraints, and $T T C_{K}$ is the maximum amount of power transfer under the contingency $K$.

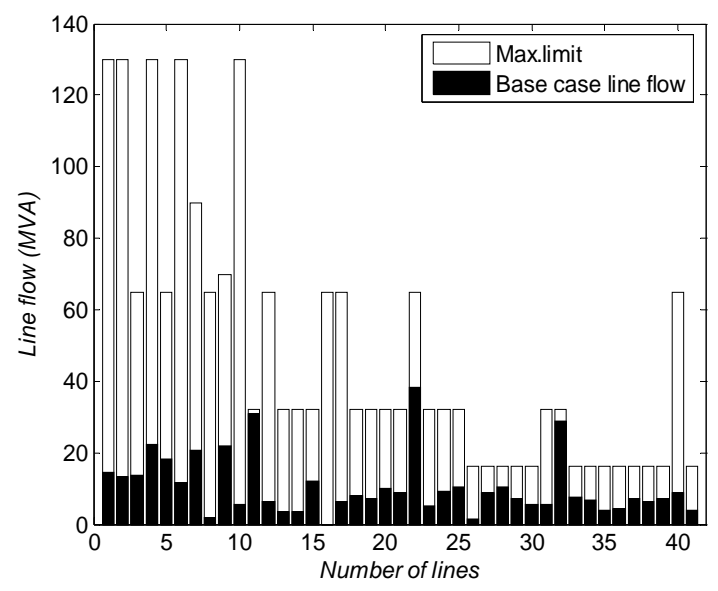

Figure 1. Base case line flows

The detailed algorithm of calculating TTC using Differential evolution is given in the flow chart as shown in the figure 2 .

From NERC's definition (NERC 1995, 1996) TRM is the transfer capability that is reserved for any uncertainties that may occur in the near future. TRM can be calculated by deterministic and probabilistic methods (NERC 1995, 1996, Sauer 1998, Yan Ou et al 2002). In this work, TRM is included by introducing single and multiline outages. In addition, for the first time, a three phase to ground fault near a bus is considered which is cleared after certain time interval with the isolation of the faulty line.

\section{Case Study and Results}

The modified IEEE-30 bus system shown in figure 3 is used as a test system (Shabaan et al, 2003). The system has three areas with two generators in each area. We have calculated TTC considering the transfer of power from area 2 to area 1 and power transfer of area 2 to area 3. For both cases, TTC is calculated with and without considering TRM. To verify the validity of the proposed algorithm, the results are compared with those obtained using PSO method and with other published results. The generation capacities $(M W)$ and real power demand $(M W)$ of each area are given in the Appendix (Table A). Simulation parameters of the two methods are given in Appendix (Table B).

\subsection{Calculation of TTC without considering TRM:}

\subsubsection{Transfer of power from area 2 to 1 :}

Power transfer between areas is carried out using Differential Evolution in which the generation of area 2 is varied from the base case values of 56.2 MW to maximum value of 70.0MW and at the same time the load of area 1 is also varied randomly. The active loading vector of area 1 before and after this transaction is shown in the Table 1 . It is found that the generation of area 2 reaches its maximum limit when the load of area 1 is $105.2503 \mathrm{MW}$ which is the needed value for TTC. Figure 4 shows the convergence 
characteristics of TTC using DE and PSO. It can be seen that DE gives better optimal results. The CPU time needed for DE and PSO are 31.6057 and 43.8154 seconds respectively for the above case.

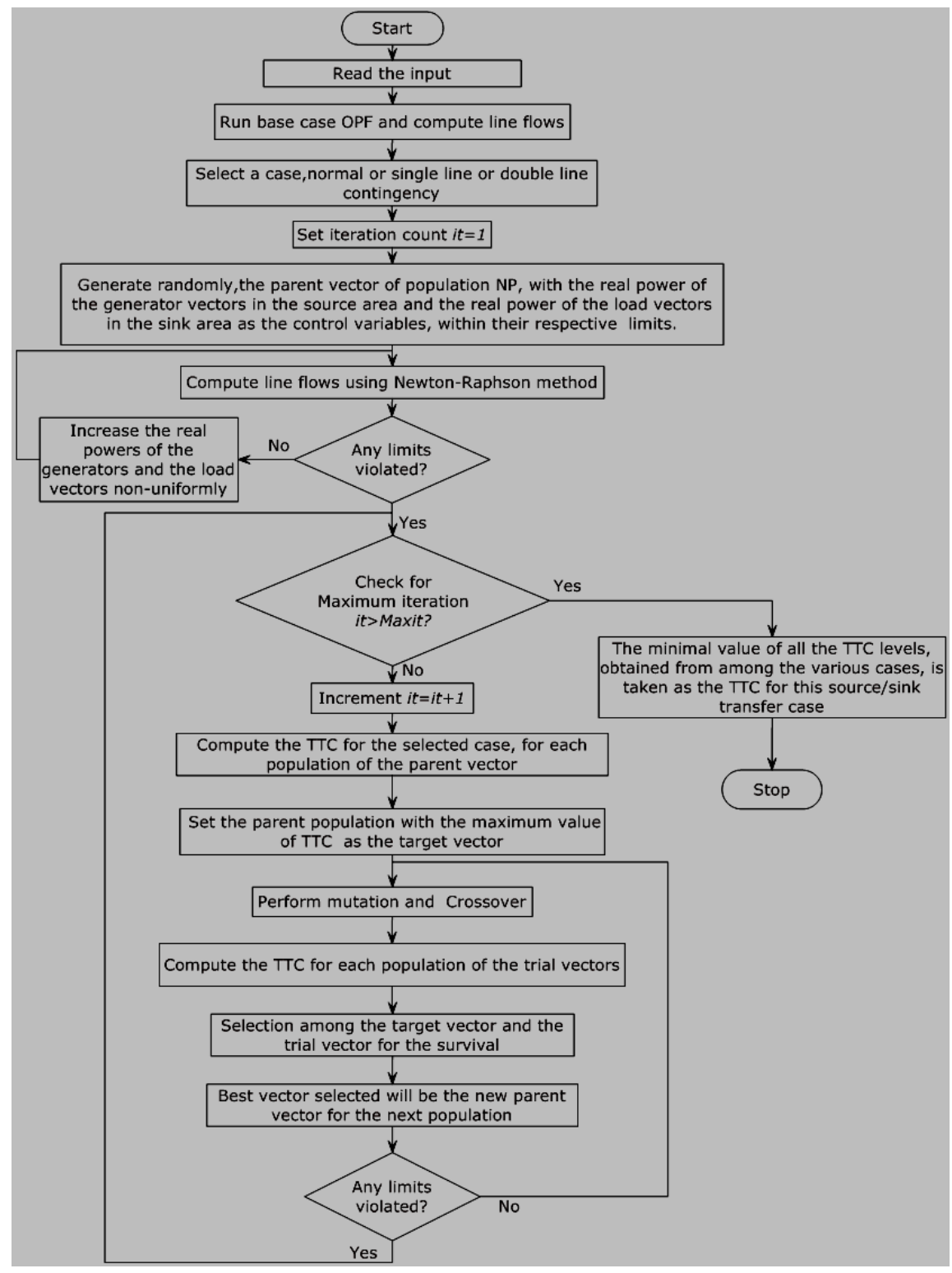

Figure 2. Flowchart for calculating TTC including contingency 


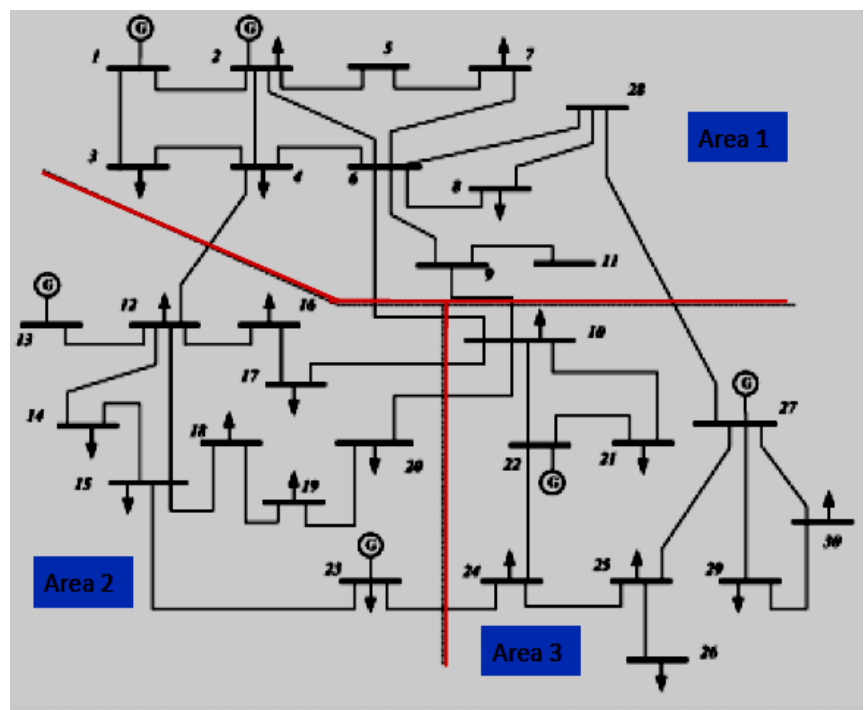

Figure 3. Modified IEEE 30 bus system

Table 1. Active loading of area $1(M W)$

\begin{tabular}{|c|c|c|c|c|c|}
\hline Load bus numbers & 2 & 3 & 4 & 7 & 8 \\
\hline Base case load $(M W)$ & 21.7 & 2.4 & 7.6 & 22.8 & 30.0 \\
\hline Increased Load using DE $(M W)$ & 33.0 & 2.9122 & 7.7439 & 30.0 & 31.7409 \\
\hline
\end{tabular}

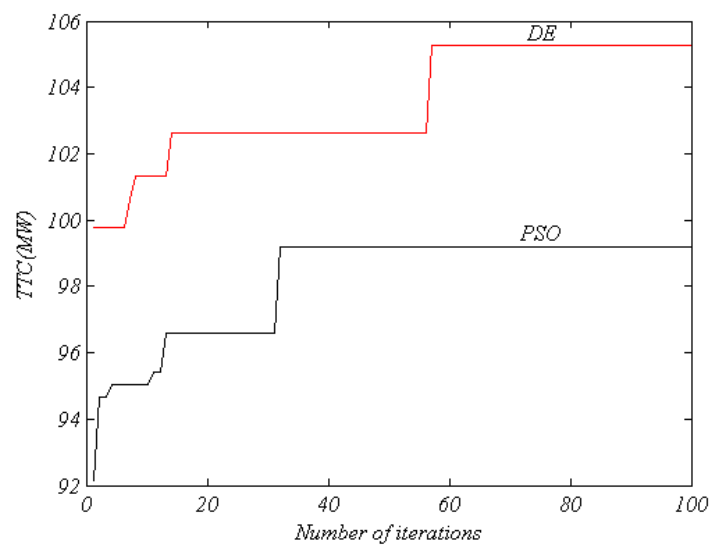

Figure 4. Convergence characteristics of TTC using DE for the transaction from area 2 to area 1

\subsubsection{Transfer of power from area 2 to 3 :}

Using DE, generation of area 2 is randomly changed between the base case values of $56.2 \mathrm{MW}$ to maximum value of $70.0 \mathrm{MW}$, along with load of area 3. We obtained the value of TTC as $65.3461 \mathrm{MW}$ for this case. The active loading vector of area 3 before and after transaction is shown in Figure 5.The TTC obtained by CPF (Shabaan et al, 2003) is $51.5 \mathrm{MW}$, due to the overload of line 6-8 and that by EP (Weerakon Onsgakul et al, 2004) is 62.65 MW where the limiting condition is the generation upper limits of area 2. The comparative results are shown in the Table 2. It is found that in both the cases of power transfer (2-1 and 2-3) TTC values given by DE are $5.80 \%$ and $3.50 \%$ greater than that obtained using PSO. This shows the robustness of DE than PSO. Such a better estimation of the TTC value helps the system operator to enhance the utilization of system capability for further operation and planning of the transmission network. Further, the CPU time required for DE and PSO are found to be 33.5843 and 44.5734 seconds respectively for the above case (2-3). Hence the proposed DE algorithm works very well in determining TTC between different areas. 


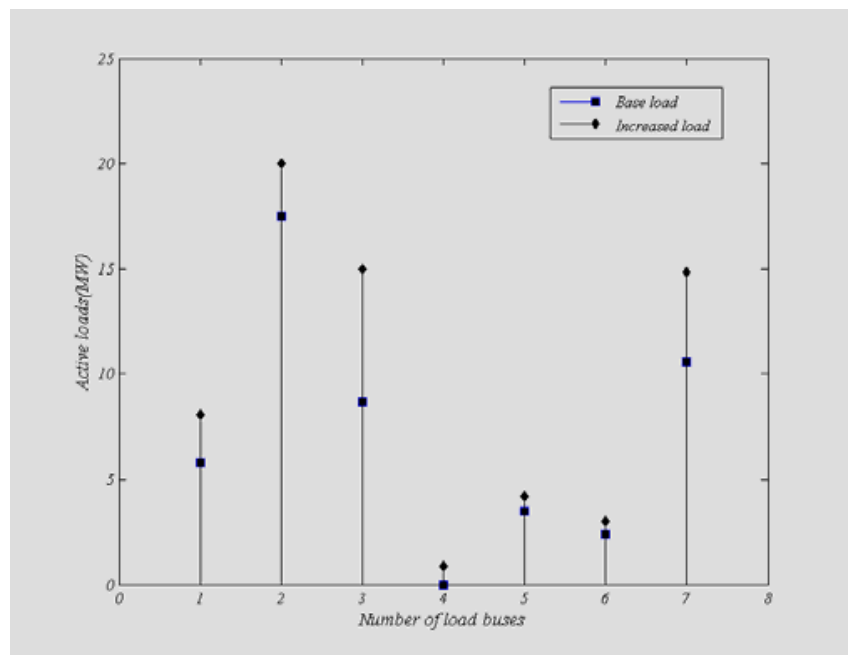

Figure 5: Base and Increased loads of area 3

Table 2. Comparison of results

\begin{tabular}{|c|c|c|c|c|c|}
\hline \multicolumn{2}{|c|}{ Area of transfer } & \multicolumn{4}{|c|}{ Total Transfer Capability $(M W)$} \\
\hline FROM & TO & $\begin{array}{c}\text { CPF[Shabaan et } \\
\text { al, 2003] }\end{array}$ & $\begin{array}{c}\text { EP [Ongsakul et } \\
a l, 2004]\end{array}$ & PSO* & $\mathrm{DE}^{*}$ \\
\hline 2 & 1 & $\begin{array}{c}\mathbf{8 8 . 5 0} \\
(6-8)\end{array}$ & $\begin{array}{c}\mathbf{9 8 . 7 7} \\
\text { (Generation } \\
\text { Of area 2) }\end{array}$ & $\begin{array}{c}99.172 \\
(6-8)\end{array}$ & $\begin{array}{c}\mathbf{1 0 5 . 2 5 0 3} \\
\text { (Generation } \\
\text { Of area 2) }\end{array}$ \\
\hline 2 & 3 & $\begin{array}{l}\mathbf{5 1 . 5 0} \\
(6-8)\end{array}$ & $\begin{array}{c}\mathbf{6 2 . 6 5} \\
\text { (Generation } \\
\text { Of area 2) }\end{array}$ & $\begin{array}{c}\text { 63.0951 } \\
(6-8)\end{array}$ & $\begin{array}{c}\mathbf{6 5 . 3 4 6 1} \\
\text { (Generator } \\
\text { Of area 2) }\end{array}$ \\
\hline
\end{tabular}

\subsection{Calculation of TTC with TRM:}

\subsubsection{Transfer of power from area 2 to 1 :}

TTC including contingencies which involves single and multi line outages is carried out for this case. The tie line between the area 2 and area $1(4-12)$, and transmission lines (5-7) \& (14-15) of area 1 and area 2, respectively are made out of service individually, and simultaneously. The outage of the line 5-7 is taken as the critical contingency which gives the least value of 99.0897MW with the line 6-8 exceeding its maximum limits as shown in the figure 6.

\subsubsection{Transfer of power from area 2 to 3 :}

The tie lines between area 2 and area 3 (10-17, $10-20$ and 23-24), are made out of service individually and multi line contingency is introduced by the outage of one of the tie line 10-17 and the line14-15 of area 2 simultaneously. The TTC value obtained for each transfer case, their respective line flows (MVA), and limiting conditions are given in Table 3. It is inferred, that the value of TTC obtained for the above case is found to be 58.1763MW, after the outage of tie line (23-24). Figure 7 shows convergence characteristics of DE for the above case. Figure 8 shows the line flows in MVA for the transaction from area 2 to area 3 using DE. Lines 11(6-8), 27(15-18) and 34(21-22) are the violated lines corresponding to the respective contingencies as shown in figure 8. The variation of real power loads in the sink area (area 3) with respect to the base case values is shown in Figure 9.

Table 3. TTC after including contingency for area 2-3

\begin{tabular}{|c|c|c|}
\hline Cases & TTC $(M W)$ & Limiting condition \\
\hline Normal & 65.3461 & Gen. of area 2 \\
\hline $10-17$ & 76.0019 & $6-8$ \\
\hline $10-20$ & 60.7472 & $15-18$ \\
\hline $\mathbf{2 3 - 2 4}$ & $\mathbf{5 8 . 1 7 6 3}$ & $\mathbf{2 1 - 2 2}$ \\
\hline $10-17 \& 14-15$ & 66.7877 & $6-8$ \\
\hline
\end{tabular}




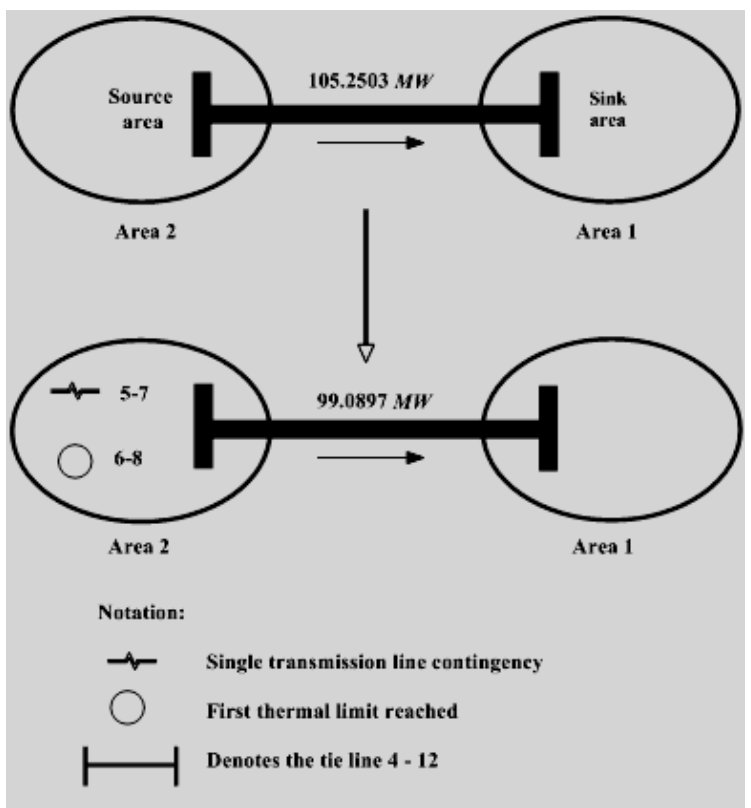

Figure 6. TTC calculation for the transaction from area 2 to 1 including contingencies

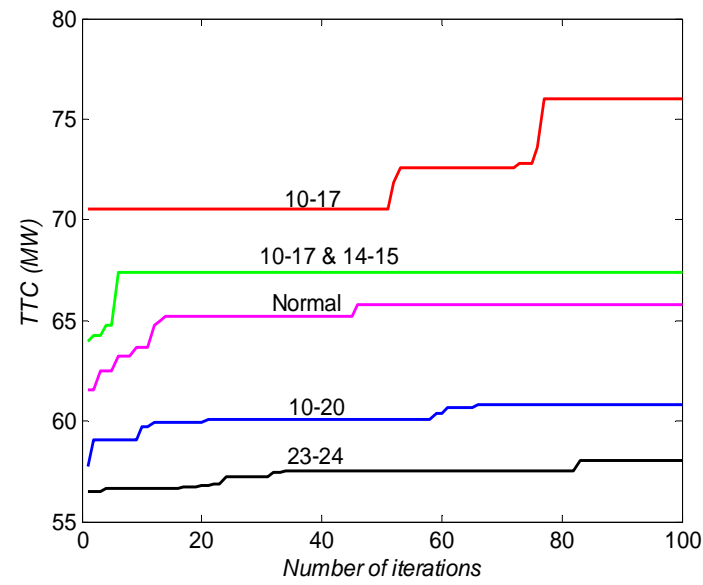

Figure 7. Convergence characteristics of TTC including the various contingencies for area 2-3 using DE

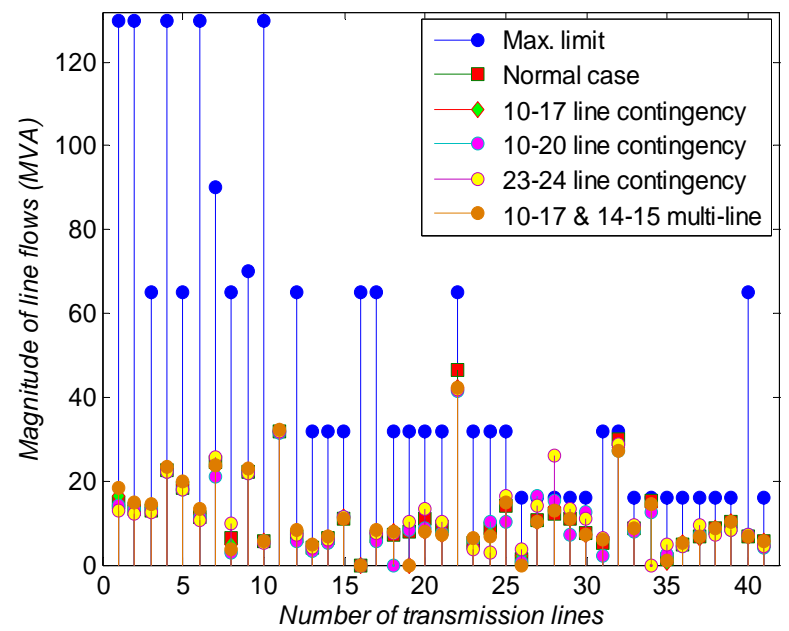

Figure 8. Line flows (MVA) for the transaction from area 2-3 using DE 


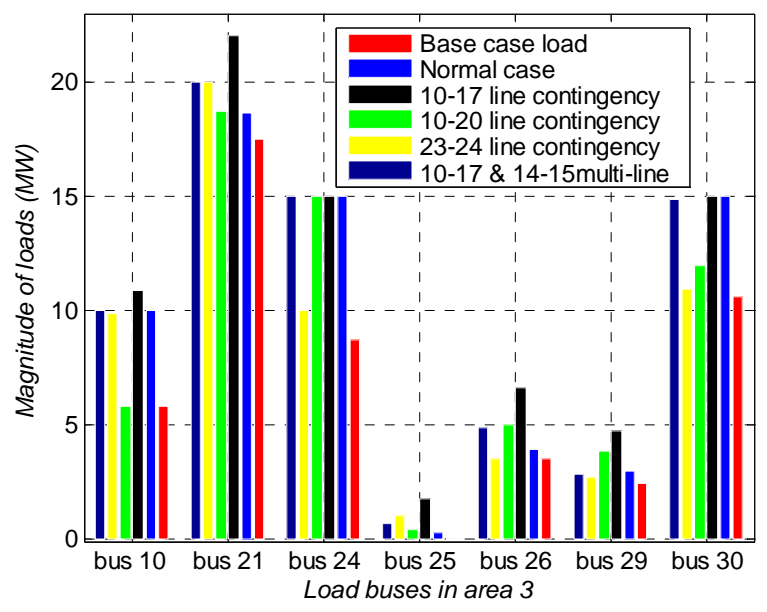

Figure 9. Variation of loads for the transaction from area 2 to area 3 using DE

5.2.3 Calculation of TTC with three phase to ground fault:

A three phase to ground fault is introduced in the transmission line connected between buses 10 \&17. Due to the fault, the generator rotor angles swing between their limits and may result in system instability. Here, the fault is subsequently cleared by isolating the faulty line from the system after $50 \mathrm{~ms}$. Rotor angle characteristics of each generator of the system are shown in the figure 10. It can be seen that rotor angles are within the safer limit and hence the system security is ensured. The value of TTC including three phase to ground fault for the area 2 to area 3 and from area 2 to area 1 is found to be $56.3531 M W$ and $97.8425 M W$ respectively.

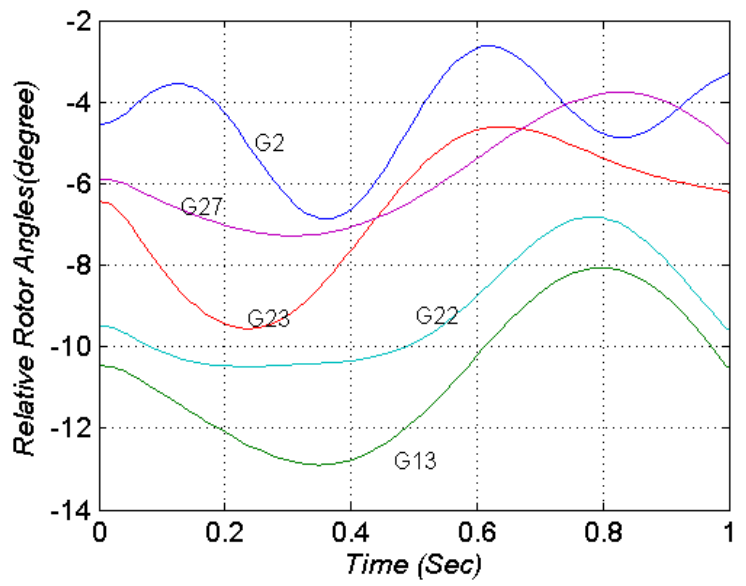

Figure 10. Generator relative rotor angle curves

\section{Conclusion}

This paper presents a detailed formulation and implementation of the OPF- based TTC calculation with Transmission Reliability Margin using Differential evolution for the first time. The algorithm is tested on the modified IEEE 30 bus system. Computer results show that the proposed method is very effective with good convergence characteristics as well compared to other optimization method called particle swarm optimization method, in determining the TTC between the different areas subjected to the system operation limits. Also, the results obtained by DE are better than the previously used methods like CPF (Shabaan et al, 2003) \& EP (Weerakon Ongsakul et al, 2004) [Table 2] and the CPU time needed for convergence is less. The accuracy of TTC is improved by considering TRM in the calculation. Also it is concluded that the total transfer capability is less when the margins are considered. 


\section{References}

Abido M. A., 2002. Optimal Power Flow using Tabu Search algorithm, Electric Power Components and Systems, Vol. 30, No. 5, pp. $469-483$.

Bakirtzis A. G., Biskas P. N., Zoumas C. E., and Petridis, V., 2002. Optimal power flow by enhanced genetic algorithm, IEEE Transactions on Power Systems, Vol. 17, No. 2, pp. 229-236.

Dai Y., McCalley J. D, and Vimal V., 2000. Simplification expansion and enhancement of direct interior point algorithm for power system maximum loadability, IEEE Transactions on Power Systems, Vol. 15, No 3, pp. 1014-1021.

Ejebe G. C., Tong J., Waight J. G., Frame J. G., Wang X., Tinney W. F., 1998. Available transfer capability calculation, IEEE Transaction on Power Systems, Vol. 13, No. 4, pp. 1521-1527.

Ejebe G. C., Waight J. G., Manuel S. N., and. Tinney W. F., 2000. Fast Calculation of linear available transfer capability, IEEE Transactions on Power Systems, Vol 15, No. 3, pp. 1112-116.

Granevner M. H., and Nwankpa C., 1999. Available transfer capability and first order sensitivity, IEEE Transaction on Power Systems, Vol. 14, No. 2, pp. 512-518.

Kennedy J., and Eberhart R. C .,1995. Particle swarm optimization, in proceedings of the 1995 IEEE International conference on Neural Networks, Vol. 4, pp. 1942-1948.

Kulworawanichpong T.,and Sujitorn S., 2002. Optimal Power Flow using Tabu Search, IEEE power Engineering Review, Vol. 22, pp. $37-40$.

Momoh J. A., El-Hawart M. E., and Adapa R., 1999. A review of selected optimal power flow literature to 1993. Part I: Non linear and quadratic programming approaches, IEEE Transaction on Power Systems, Vol. 14, No.1 pp. $105-111$.

Momoh J. A., El-Hawart M. E., and Adapa R., 1999. A review of selected optimal power flow literature to 1993. Part II: Newton, linear programming and interior point methods, IEEE Transaction on Power Systems, Vol. 14, No.1 pp. $105-111$.

North American Electric Reliability Council (NERC), 1995. Transfer Capability Definitions and Determination, NERC Report.

North American Electric Reliability Council, 1996. Available Transfer Capability Definitions and Determination: A Framework for Determining Available Transfer Capabilities of the Interconnected Transmission Networks for a Commercially Viable Electricity Market, NERC Report.

Park J. B., 2005. A Particle swarm optimization for Economic Dispatch with Non-smooth cost functions, IEEE Transactions on Power systems, Vol. 20, No. 1, pp. 34-42.

Price K., 1996. Differential Evolution: A Fast and simple Numerical optimizer, Biennial conference of the North American Fuzzy Information Processing society, pp. 524 - 527.

Price K., and Storn M., 1999. An introduction to Differential Evolution, New optimization, (Eds) D. Corne, M. Dorigo et al., London, McGraw Hill International (UK) Limited, pp. 79 -108.

Rajathy R., Gnanadass R., Manivannan K., and Harish Kumar, 2009. Risk- constrained optimal bidding strategies using Differential evolution, International Journal of Power, Energy, Automation and Intelligence, Vol. 2 No. 1, pp. 96-103.

Roa-Sepulveda C. A., and Lazo B. J., 2003. A Solution to the Optimal Power Flow using Simulated Annealing, Electrical Power and Energy Systems, Vol. 25, No. 1, pp. $47-57$.

Sauer P. W., 1998. Alternatives for calculating Transmission Reliability Margin in ATC calculation, $31^{\text {st }}$ Hawaii International Conference System Science, Vol. 3, p. 89.

Shaaban M., Ni Y., Dai H., and Wu F. F., 2003. Calculation of total transfer capability incorporating the effect of reactive power, Electric Power Systems Research, Vol. 64, No. 3, pp. 181-188.

Yan Ou, Chanan Singh, 2002. Assessment of Available Transfer capability and margins, IEEE Transaction on Power Systems, Vol. 17, No. 2, pp. 463-468.

Yuryevich J.,and. Wong K. P, 1999. Evolutionary programming based optimal power flow algorithm, IEEE Transactions on Power Systems, Vol. 14, No. 4, pp. 1245-1250.

Weerakorn Ongsakul and Peerapol Jirapong, 2004. Calculation of Total Transfer Capability by Evolutionary programming, Tencon 2004, IEEE Region 10 conference, Vol. 3, pp. 492-495.

\section{Appendix}

Table A. Generation and load of each area

\begin{tabular}{|c|c|c|}
\hline Area & Total Generation $(M W)$ & Load $(M W)$ \\
\hline 1 & 160 & 84.5 \\
\hline 2 & 70 & 56.2 \\
\hline 3 & 105 & 48.5 \\
\hline
\end{tabular}


Table B. Simulation parameters of the proposed methods

\begin{tabular}{|l|c|c|}
\hline Parameters & PSO & DE \\
\hline Population size $\left(N_{p}\right)$ & 40 & 40 \\
\hline$C_{1}$ & 2 & - \\
\hline$C_{2}$ & 2 & - \\
\hline$W_{\max }$ & 0.9 & - \\
\hline$W_{\min }$ & 0.4 & - \\
\hline Cross-over constant $\left(C_{R}\right)$ & - & 0.8 \\
\hline Scaling Factor $(F)$ & - & 0.55 \\
\hline Maximum iteration & 100 & 100 \\
\hline
\end{tabular}

\section{Biographical notes}

R. Rajathy obtained her B.E in Electrical and Electronics Engineering and M. E in power system with Distinction from Thiagarajar College of Engineering, Madurai and now with the Department of Electrical and Electronics Engineering, Pondicherry Engineering College, Pondicherry, India and currently pursuing for Ph. D. Her special fields of interest are Power System Optimization and Power System Restructuring.

Gnanadass Ramachandran received the Undergraduate Degree in Electrical Engineering and the Masters degree in Power Systems Engineering with Distinction in 1991 and 1993, respectively. He has obtained the Ph.D. degree in the Department of Electrical \& Electronics Engineering, Pondicherry Engineering College, Pondicherry, India in July 2005. He is working as a teaching faculty in Pondicherry Engineering College since 1996. He successfully completed his post doctoral fellowship at Department of Electrical and Computer Engineering, Iowa State University, Ames, USA under BOYSCAST fellowship sponsored by Department of Science and Technology, Government of India. His field of interest is power system privatization, reactive power pricing and management, voltage stability, concepts of power system restructuring and optimization problems.

K. Manivannan obtained his Ph. D. from Indian Institute of Madras, India and now working as a faculty in the Department of Electrical \& Electronics Engineering, Pondicherry Engineering College, Pondicherry, India. His special fields of interest are Digital Signal Processing, and Optimization Techniques.

Harish Kumar obtained his Ph. D. from Mangalore University, India and now working as a faculty in the Department of Physics, Pondicherry Engineering College, Pondicherry, INDIA. His special fields of interest are Computational and Theoretical Physics, and Optimization Techniques.

Received November 2009

Accepted March 2010

Final acceptance in revised form June 2010 